\title{
Stalking - Aktuelle Entwicklungen und Tendenzen zur Schaffung eines speziellen Tatbestandes ${ }^{1}$
}

\section{A. Einführung}

Seit einigen Jahren beschäftigt die Strafverfolgungsbehörden ein neues Phänomen, das systematisch-zielgerichtete Nachstellen und Verfolgen von Personen (»Stalking«). Es handelt sich um ein in unterschiedlichsten Formen auftretendes Erscheinungsbild, das mittlerweile als Verhaltensweise mit erheblichen Beeinträchtigungen für das Opfer und daher mit strafrechtlicher Bedeutung erkannt worden ist. Das Phänomen Stalking ist in den USA und Großbritannien bereits seit Anfang der neunziger Jahre Gegenstand kriminologischer Forschung und auch Inhalt strafrechtlicher Gesetze, ${ }^{2}$ während das Stalking in Deutschland erst in jüngster Zeit Aufmerksamkeit gefunden hat $^{3}$ und die Relevanz des Phänomens erst allmählich bewusst wird. ${ }^{4}$

Einen ersten Versuch zur Verbesserung der Situation der Opfer wurde durch das Gewaltschutzgesetz ${ }^{5}$ unternommen. Insgesamt bereitet das Phänomen Stalking erhebliche Schwierigkeiten und dringt zunehmend in das Bewusstsein der Öffentlichkeit vor. Aktuell beschäftigt sich der Gesetzgeber erneut mit dem Problem.

\section{Terminologie}

Der Begriff Stalking kommt aus der englischen Jägersprache und wurde ursprünglich von Jägern für das Heranpirschen und Einkreisen der Beute, des verfolgten Wildes benutzt. ${ }^{6}$ Nach neuerem Sprachverständnis wird mit dem Begriff Stalking auch das

1 Dr. Sascha Vander ist wissenschaftlicher Mitarbeiter am Lehrstuhl für Bürgerliches Recht und Gewerblichen Rechtsschutz der Heinrich-Heine-Universität Düsseldorf bei Prof. Dr. Jan Busche.

2 In der Zwischenzeit wurden auch in Kanada, Australien, Japan, Belgien und den Niederlanden gesonderte Straftatbestände geschaffen. Auch Österreich erwägt die Einführung eines solchen Tatbestandes.

3 Löbmann, MschrKrim 2002, 25; Gropp, NK 2002, 112. Ausführlich zur strafrechtlichen Situation in England: von Pechstaedt, Stalking, Strafbarkeit nach englischem und deutschem Recht, 1999, S. 69 ff. Eine Übersicht zu aktuellen Forschungsprojekten bietet Bettermann, in: Bettermann/Feenders, Stalking, Grenzen und Möglichkeiten der Intervention, 2004, S. 3, 9. Vgl. auch die Untersuchungen zum Stalking in Fällen häuslicher Gewalt von Löbmann, in: Bettermann/Feenders [Fn. 3] S. 75 ff; zum Prominenten-Stalking vgl. Hoffmann, in: Bettermann/Feenders [Fn. 3], S. $101 \mathrm{ff}$.

4 Hoffmann, Kriminalistik 2001, 34; vgl. Rinio, Kriminalistik 2002, 531.

5 Gesetz zum zivilrechtlichen Schutz vor Gewalttaten und Nachstellungen vom 11.12.2001 (GewSchG), BGBl. I 2001, 3513.

6 Englisch »to stalk « = anpirschen, anschleichen; »stalker « = Pirschjäger. Im Allgemeinen wird das englische Verb »to stalk « übersetzt mit »belästigen«. Ausführlich zum Begriff des Stalking: von Pechstaedt [Fn. 3], S. 18 ff. 
zwanghafte Verfolgen und Belästigen einer anderen Person verbunden, während demgegenüber in der deutschen Sprache kein begriffliches Pendant existiert. ${ }^{7}$

Eine klare juristische Definition des Begriffs Stalking besteht nicht ${ }^{8}$ und bereitet deutliche Probleme, da sich das Stalking nicht auf ein bestimmtes Verhaltensmuster reduzieren lässt, sondern seine Besonderheit gerade in den mannigfaltigen Erscheinungsformen und Motiven findet. Teilweise wird Stalking als böswilliges und absichtliches Verfolgen und Belästigen einer anderen Person, das deren Sicherheit bedroht, bezeichnet. ${ }^{9}$ Da die Motive des Täters vielfach nicht ergründbar sind, erscheint es sinnvoll und praktikabler, eine Definition objektiv zu halten und auf das günstigstenfalls sichtbare und daher beschreibbare Verhalten des Täters abzustellen. ${ }^{10}$ Stalking könnte demnach definiert werden als die systematische und exzessive Belästigung und Verfolgung einer Einzelperson aus innerpsychischen Gründen. ${ }^{11}$ Objektiv gesehen ist Stalking - vereinfacht dargestellt - eine fortgesetzte Behelligung des Opfers gegen dessen Willen durch direkte oder indirekte Kontaktaufnahme. ${ }^{12}$

\section{Erscheinungsformen und Auswirkungen}

Zu den häufigsten Stalking-Handlungen gehören Telefonterror, Überwachung und Beobachtung, demonstrative ständige Anwesenheit, Verfolgung, Annäherung und unerwünschte Kommunikationsversuche, Drohungen und Bedrohung, körperliche Übergriffe, Ehrverletzungen, Zusendung bzw. Hinterlassen bestimmter Mitteilungen oder Gegenstände sowie Vermögens- und Eigentumsschädigungen. ${ }^{13}$ Die Nennung dieser Verhaltensweisen veranschaulicht, weshalb Stalking auch als spezifisches Machtspiel verstanden wird, das auf die Gewinnung von Kontrolle über das Opfer abzielt. ${ }^{14}$

Charakteristisch ist dabei, dass viele zum Teil stark heterogene Einzelhandlungen erst durch ihre Wiederholung und ihre Kombination zum Stalking werden. ${ }^{15}$ So können für sich allein genommen wenig bedrohliche Ereignisse wie das Zusenden eines Briefes durch ständige Wiederholung zermürbenden und bedrohlichen Charakter annehmen. ${ }^{16}$

Die Belästigungen finden in den meisten Fällen zunächst subtil statt. Für die Betroffenen wird jedoch aus der anfänglichen Nerverei sehr schnell eine Belästigung, aus Belästigungen werden Beeinträchtigungen des täglichen Lebens, und die Beeinträchti-

7 Kerbein/Pröbsting, ZRP 2002, 76, 77; Schäfer, Kriminalistik 2000, 587.

8 Meyer, ZStW 115 (2003), 249; vgl. Rinio, Kriminalistik 2002, 531, der darauf hinweist, dass eine Definition auch durch das Gewaltschutzgesetz nicht eingeführt wurde.

9 Vgl. Goebel/Lapp, Kriminalistik 2003, 369.

10 von Pechstaedt, Polizei und Wissenschaft 2002, 45, 46.

11 Knecht, Kriminologie 2003, 364.

12 von Pechstaedt, Polizei und Wissenschaft 2002, 45, 46.

$13 \mathrm{Zu}$ den Einzelheiten vgl. von Pechstaedt [Fn. 3], S. 30 ff; vgl. auch Knecht, ArchKrim. 2003, 1, 5 .

14 Meyer, ZStW 115 (2003), 249, 252 (m. w N).

15 Gropp, NK 2002, 112; Löbmann, MschrKrim 2002, 25, 27; Meyer, ZStW 115 (2003), 249, 251.

16 Löbmann, MschrKrim 2002, 25, 27. 
gungen werden schließlich zu Psychoterror. ${ }^{17}$ Rund $70 \%$ der Opfer fühlen sich dazu gezwungen, ihre Lebensumstände zu ändern. ${ }^{18}$ Einschlägige Untersuchungen bestätigen zudem abstrakt, dass schweres Stalking krank macht. ${ }^{19}$

Die Motivationslage von Stalkern ist ebenso unterschiedlich wie die zu beobachtenden Verhaltensweisen und daher kaum verallgemeinerungsfähig. In der Psychologie ${ }^{20}$ wird vor diesem Hintergrund mit Fallgruppen gearbeitet, um eine Klassifikation von Stalkern vornehmen zu können. Dabei reichen die Motive von enttäuschter Zuneigung bis hin zu Rachegelüsten für vermeintlich erlittenes Unrecht. ${ }^{21}$

\section{Praktische Bedeutung}

Die praktische Bedeutung des Stalking ist nicht zu unterschätzen. In das Bewusstsein der Öffentlichkeit gelangen regelmäßig nur solche Fälle, in denen Prominente Opfer von Stalkern werden. Die Mehrheit der Fälle geht jedoch völlig unter. Eine amerikanische Studie belegt, dass 8\% der amerikanischen Frauen und 2\% der amerikanischen Männer in ihrem Leben schon einmal gestalkt worden sind, wobei die Dunkelziffer wesentlich höher liegen dürfte. ${ }^{22}$ In den überwiegenden Stalking-Fällen (ca. 60\% der Fälle) treten als Täter ein Mann und als Opfer eine Frau in Erscheinung, wohingegen der umgekehrte Fall lediglich halb so oft vorkommt. ${ }^{23}$ Die Dauer des Stalking-Verhaltens kann beträchtlich sein und lag in einer amerikanischen Repräsentativstudie bei 1,8 Jahren. ${ }^{24}$

Für Deutschland liegen bislang kaum Zahlen aus abgeschlossenen empirischen Untersuchungen über die Erscheinungsformen und Auswirkungen des Stalking vor, ${ }^{25}$ so dass überwiegend auf ausländische Erfahrungen und Erkenntnisse zurückzugreifen ist. ${ }^{26}$ Neben einer umfassenderen Studie des Zentralinstituts für Seelische Gesundheit in Mannheim aus dem Jahre $2004^{27}$ ist jedoch insbesondere auf eine aktuelle, zurzeit

17 Pollähne, NK 2002, 56. Wondrak, in: Bettermann/Feenders [Fn. 3], S. 21 spricht insoweit von einer »eskalierenden Dynamik «.

18 Löbmann, MschrKrim 2002, 25, 29.

19 So das klare Ergebnis der Studie der TU Darmstadt vom Frühjahr diesen Jahres. Hierzu: http://www.weisser-ring.de/bundesgeschaeftsstelle/aktuell/meldungen/ stalking_studie_der_tu_darmstadt/index.php.

20 Allgemein zur Psychologie des Stalking: Voß, in: Bettermann/Feenders [Fn. 3], S. 37 ff.; vgl. auch Kobbé, in: Bettermann/Feenders [Fn. 3], S. $251 \mathrm{ff}$.

21 Eingehend zur Klassifizierung bestimmter Hauptfallgruppen: Mullen/MacKenzie, in: Bettermann/Feenders [Fn. 3], S. 51, 55 f.; vgl. auch Knecht, ArchKrim. 2003, 1, 5; von Pechstaedt, Polizei und Wissenschaft 2002, 45, 47; ders. [Fn. 3], S. 44 ff.

22 Vgl. Borchert, FPR 2004, 239; Meyer, ZStW 115(2003), 249, 256; vgl. auch Löbmann, MschrKrim 2002, 25, 27, die die hohe Dunkelziffer mit der mangelnden Anzeigebereitschaft der Opfer begründet, die lediglich bei ca. 50\% liegen soll.

23 von Pechstaedt, Polizei und Wissenschaft 2004, 45, 47. Vgl. auch den Bericht über männliche Opfer von Lenz, in: Bettermann/Feenders [Fn. 3], S. 273 ff. Zu Stalking in lesbischen Beziehungen: Ohms, in: Bettermann/Feenders [Fn. 3], S. $121 \mathrm{ff}$.

24 Löbmann, MschrKrim 2002, 25, 28; Meyer, ZStW 115(2003), 249, 257.

25 Meyer, ZStW 115(2003), 249, 251, 254; von Pechstaedt [Fn. 3], S. 64.

26 Ausführlich zu statistischen Erhebungen zum Stalking: von Pechstaedt [Fn. 3], S. 49 ff.

27 Die Ergebnisse der Studie sind abrufbar unter: http://www.zi-mannheim.de/fileadmin/ user_upload/pdfdateien/pressemitteilungen/pressemitteilungen04/Stalking.pdf. 
in der Veröffentlichung befindliche Studie der TU Darmstadt hinzuweisen, an deren Arbeitsstelle für forensische Psychologie ${ }^{28}$ im Zeitraum von 2002 bis 2005 die bisher größte wissenschaftliche Studie zum Thema Stalking im deutschsprachigen Raum erstellt wurde. ${ }^{29}$ Die bislang veröffentlichten Teilergebnisse der Studie ${ }^{30}$ decken sich weitgehend mit den Erkenntnissen ausländischer Studien, wobei jedoch die ermittelte längere Dauer des Stalking (28 Monate) sowie der deutlich höhere Anteil männlicher Täter $(81 \%)$ hervorzuheben sind.

\section{B. Strafrechtliche Bewertung}

Die strafrechtliche Bewertung des Stalking fällt nicht leicht. Bei der Zuordnung der Verhaltensweisen und ihrer Auswirkungen zu einzelnen Tatbeständen erfährt man keine Hilfe durch die Kommentar- und Lehrbuchliteratur und auch in der obergerichtlichen Rechtsprechung existieren bislang praktisch keine Entscheidungen zu diesem Bereich. ${ }^{31}$ Allein von Pechstaedt hat sich in seiner Dissertation aus dem Jahre 1999 mit der strafrechtlichen Bewertung des Stalking intensiv auseinandergesetzt. ${ }^{32}$

\section{Einschlägige Straftatbestände}

Fraglich ist, ob die Verhaltensweisen bzw. Verhaltensmuster von Stalkern nach der gegenwärtigen Gesetzeslage strafbar sind.

\section{Einzelhandlungen}

Einzelne Handlungen können strafbar sein als Beleidigung in allen Formen der $\S \S 185$ ff. StGB, Körperverletzungsdelikt nach den $\S \S 223 \mathrm{ff}$. StGB, Nötigung nach $\S 240$ StGB, Bedrohung nach § 241 StGB, Freiheitsberaubung nach § 239 StGB, Hausfriedensbruch nach $\S 123$ StGB, Sachbeschädigung nach $\S 303$ StGB sowie im Falle einer Eskalation auch als schwerwiegendes Gewalt- oder Tötungsdelikt.

Eine Vielzahl der Stalking-Handlungen (z. B. gehäufte Telefonanrufe, Auflauern vor der Wohnung oder dem Arbeitsplatz, etc.) erfasst das deutsche Strafrecht - abgesehen von den Möglichkeiten nach dem $\mathrm{GewSchG}^{33}$ - hingegen nicht.

28 http://www.tu-darmstadt.de/fb/fb3/psy/diffpsy/index.htm. Aktuelle Informationen und Berichte über die Arbeitsgruppe sind im Internet abrufbar unter: http://www.stalkingforschung.de (Rubrik: aktuelles).

29 Informationen und Hinweise zu diesem Forschungsprojekt sind im Internet unter http:// www.stalkingforschung.de abrufbar.

30 Die Studie wurde durch den Weißen Ring gefördert. Auf der Website des Weißen Rings bietet dieser erste Studienergebnisse: http://www.weisser-ring.de/bundesgeschaeftsstelle/aktuell/meldungen/stalking_studie_der_tu_darmstadt/index.php.

31 Meyer, ZStW 115 (2003), 249, 259.

32 von Pechstaedt [Fn. 3], S. 113 ff. Hinzweisen ist auch auf die jüngere Untersuchung von Meyer, ZStW 115 (2003), 249 ff.

33 Hierzu: B I 2b. 


\section{Gesamtverhalten}

Während die Strafbarkeit von Einzelhandlungen noch relativ einfach zu beurteilen ist, bereitet die Beurteilung einer Strafbarkeit des Gesamtverhaltens erhebliche Schwierigkeiten, insbesondere wenn die Einzelhandlungen selbst nicht strafbar sind.

\section{a) Straftatbestände nach dem StGB}

Viele Verhaltensweisen erfüllen für sich genommen, als Einzelakt, noch nicht einen Straftatbestand des StGB und sind im Regelfall bei einmaliger Begehung noch hinzunehmen. ${ }^{34}$ Zudem haben die Opfer vielfach mit Nachweisschwierigkeiten zu kämpfen. So fällt etwa der Nachweis einer unmittelbaren Gesundheitsbeeinträchtigung als Körperverletzungserfolg regelmäßig schwer, ${ }^{35}$ auch wenn einschlägige Untersuchungen abstrakt bestätigen, dass schweres Stalking krank macht. ${ }^{36}$ In diesem Zusammenhang sei stellvertretend auf ein Urteil des OLG Düsseldorf hingewiesen, wonach nächtliche Telefonanrufe keine Körperverletzung darstellen sollen, sofern sie reine Befindlichkeitsstörungen ohne einen medizinisch bedeutsamen Krankheitswert verursachen. ${ }^{37}$ Psychovegetative Vorgänge wie Schweißausbrüche, Herzklopfen oder Durchfall, die ein plötzliches Erscheinen des Täters oder das Zusenden von Nachrichten durch diesen auslösen kann, überschreiten die Erheblichkeitsschwelle trotz ihrer Lästigkeit nicht, so dass eine Strafbarkeit nach $\S 223$ StGB ausscheidet. ${ }^{38}$ Höchstens in Fällen, in denen Nachstellungen und Bedrohungen zu massiven depressiven Verstimmungen mit Schlaf- und Konzentrationsstörungen bis hin zu Selbstmordgedanken führen, liegen neben der seelischen Erschütterung kumulativ eine körperliche Misshandlung und eine Gesundheitsbeschädigung vor. ${ }^{39}$

Auch eine nötigende oder beleidigende Qualität des Handelns der belästigenden Person bleibt in der Regel zu unbestimmt, wenn nicht einzelne Übergriffe insoweit als tatbestandsmäßig anzusehen sind. ${ }^{40}$ Eine körperliche Einwirkung auf das Opfer, die den verfassungsrechtlichen Anforderungen an die Auslegung des Gewaltbegriffs entspricht, wird meist nicht feststellbar sein - selbst dann nicht, wenn das Opfer die Drangsalierung als gegenwärtiges und körperliches Übel empfindet. ${ }^{41}$

34 Erklärung des hessischen Staatsministers Wagner, in: Plenarprotokoll des Bundesrats, Stenografischer Bericht über die 802. Sitzung, Anlage 30, S. 399 C.

35 Gesetzesantrag des Landes Hessen, BR-Drs. 551/04, S. 5 des Gesetzesentwurfs. Vgl. auch die Erklärung des hessischen Staatsministers Wagner, in: Plenarprotokoll des Bundesrats, Stenografischer Bericht über die 802. Sitzung, Anlage 30, S. 399 C.

36 So das klare Ergebnis der Studie der TU Darmstadt vom Frühjahr dieses Jahres. Hierzu: http://www.weisser-ring.de/bundesgeschaeftsstelle/aktuell/meldungen/ stalking_studie_der_tu_darmstadt/index.php.

37 OLG Düsseldorf, Beschluss vom 23.05.2002 - 2a Ss 97/02 - 41/02 II, NJW 2002, 2118.

38 Meyer, ZStW 115 (2003), 249, 261.

39 Meyer, ZStW 115 (2003), 249, 261.

40 Vgl. Gesetzesantrag des Landes Hessen, BR-Drs. 551/04, S. 5 des Gesetzesentwurfs.

41 Meyer, ZStW 115 (2003), 249, 261. 
Eine Strafbarkeit des Gesamtverhaltens nach anderen Vorschriften kommt regelmäBig ebenfalls nicht in Betracht. ${ }^{42}$

\section{b) Nebenstrafrecht}

Einen möglichen Ansatz zur strafrechtlichen Verfolgung des Stalking bietet das am 1. Januar 2002 in Kraft getretene Gewaltschutzgesetz, ${ }^{43}$ das in $\S 1$ Abs. 2 S. 1 Nr. 2 lit. b die Möglichkeit einer gerichtlichen Unterlassungsanordnung vorsieht, wenn eine Person widerrechtlich und vorsätzlich eine andere Person dadurch unzumutbar belästigt, dass sie ihr gegen den ausdrücklich erklärten Willen wiederholt nachstellt oder sie unter Verwendung von Fernkommunikationsmitteln verfolgt. Für den Fall der Fortsetzung des Nachstellens und damit der Zuwiderhandlung gegen jene gerichtliche Anordnung sieht $\S 4$ S. 1 GewSchG eine Freiheitsstrafe bis zu einem Jahr oder Geldstrafe vor.

Dies ist nichts anderes als die Strafbarkeit von Stalking, wenn auch nicht im StGB und wenn auch auf dem Umweg über die Kriminalisierung des Ungehorsams gegenüber vollstreckbaren gerichtlichen Anordnungen. ${ }^{44}$ Es besteht somit bereits nach geltendem Recht strafrechtlicher Schutz gegen Stalking. Vor Inkrafttreten des GewSchG war das Verhalten eines Stalkers, der es bei Belästigungen seines Opfers, etwa durch ständige Telefonanrufe, das unerwünschte Zusenden von Geschenken oder das Beobachten oder Verfolgen seines Opfers beließ, strafrechtlich nicht oder nur schwierig erfassbar. ${ }^{45}$

\section{Gesetzesinitiativen}

Die jüngsten in der Öffentlichkeit besonders hervorgetretenen Fälle des Stalking von Prominenten sowie einige spektakuläre Fälle mit Todesfolge haben die Debatte um eine diesbezügliche Verschärfung bzw. Erweiterung des Strafrechts angeheizt. Das Land Hessen hat die Initiative ergriffen und Mitte 2004 einen ersten Gesetzesantrag vorgelegt. ${ }^{46}$

42 Zu den Einzelheiten vgl. Meyer, ZStW 115 (2003), 249, 260 ff; von Pechstaedt [Fn. 3], S. $113 \mathrm{ff}$.

43 BGB1. I 2001, 3513.

44 Pollähne, NK 2002, 56, 58; vgl. auch Frommel, ZRP 2001, 287, 291; Grziwotz, NJW 2002, 872, 873; siehe auch Hartenbach, in: Plenarprotokoll des Bundesrats, Stenografischer Bericht über die 809. Sitzung, S. 88 B; a. A. Meyer, ZStW 115 (2003), 249, 269 f., der die Regelungen des GewSchG als zu »unsubstantiiert « erachtet; so auch von Pechstaedt, in: Bettermann/Feenders [Fn. 3], S. 147, 164, der darauf hinweist, dass durch § 4 GewSchG nicht Stalking, sondern der Ungehorsam gegen eine zivilgerichtliche Verfügung bestraft wird.

45 Gropp/von Pechstaedt, in: Bettermann/Feenders [Fn. 3], S. 169, 174; Rinio, Kriminalistik 2002, 531.

46 Gesetzesantrag des Landes Hessen, BR-Drs. 551/04. 


\section{Gesetzesentwurf des Bundesrats}

Den Gesetzesentwurf brachte das Land Hessen am 5. Juli 2004 als Gesetzesantrag in den Bundesrat ein, ${ }^{47}$ welcher auf Grundlage einer Empfehlung der federführenden Ausschüsse $^{48}$ eine in weiten Teilen modifizierte Fassung des hessischen Entwurfs in den Deutschen Bundestag einführte. ${ }^{49}$

\section{Inhalt}

Der aktuelle Gesetzesentwurf verfolgt ein zweigliedriges Regelungskonzept und setzt sowohl im materiellen als auch im Verfahrensrecht an.

\section{a) Materielle Regelungen}

Der aktuelle Gesetzesentwurf des Bundesrats verfolgt wegen der Nähe zur Freiheitsberaubung ${ }^{50}$ die Statuierung eines eigenständigen Tatbestandes in $\S 238$ StGB mit der Bezeichnung »Schwere Belästigung «: $: 51$

\section{$\S 238$ StGB EF \\ Schwere Belästigung}

(1) Wer unbefugt und in einer Weise, die geeignet ist, einen Menschen in seiner Lebensgestaltung erheblich zu beeinträchtigen, diesen nachhaltig belästigt, indem er fortgesetzt

1.ihm körperlich nachstellt oder ihn unter Verwendung von Kommunikationsmitteln verfolgt,

2.ihn, einen seiner Angehörigen oder eine andere ihm nahe stehende Person mit einem empfindlichen Übel bedroht oder

3. andere, ebenso schwerwiegende Handlungen vornimmt,

wird mit Freiheitsstrafe bis zu einem Jahr oder mit Geldstrafe bestraft.

(2) Bringt der Täter das Opfer, einen Angehörigen des Opfers oder einen anderen dem Opfer nahe stehenden Menschen durch die Tat in die Gefahr einer erheblichen Gesundheitsbeschädigung, so ist die Strafe Freiheitsstrafe von drei Monaten bis zu fünf Jahren.

47 Gesetzesantrag des Landes Hessen, Entwurf eines Strafrechtsänderungsgesetzes - Gesetz zur Bekämpfung unzumutbarer Belästigungen (»Stalking-Bekämpfungsgesetz«), BR-Drs. 551/04.

48 Empfehlungen der Ausschüsse, BR-Drs. 551/1/04.

49 Gesetzesentwurf des Bundesrats, BT-Drs. 15/5410.

$50 \mathrm{Vgl}$. Gesetzesentwurf des Bundesrats, BT-Drs. 15/5410, S. 6.

51 Gesetzesentwurf des Bundesrats, BT-Drs. 15/5410. 
(3) Auf Freiheitsstrafe von einem Jahr bis zu zehn Jahren ist zu erkennen, wenn der Täter das Opfer, einen Angehörigen des Opfers oder einen anderen dem Opfer nahe stehenden Menschen bei der Tat körperlich misshandelt oder durch die Tat in die Gefahr des Todes oder einer schweren Gesundheitsbeschädigung bringt.

(4) Verursacht der Täter durch die Tat den Tod des Opfers, eines Angehörigen des Opfers oder eines anderen dem Opfer nahe stehenden Menschen, so ist die Strafe Freiheitsstrafe nicht unter 3 Jahren.

(5) In minder schweren Fällen des Absatzes 3 ist auf Freiheitsstrafe von sechs Monaten bis zu fünf Jahren, in minder schweren Fällen des Absatzes 4 auf Freiheitsstrafe von einem Jahr bis zu zehn Jahren zu erkennen.

(6) In den Fällen des Absatzes 1 wird die Tat nur auf Antrag verfolgt, es sei denn, dass die Strafverfolgungsbehörde wegen des besonderen öffentlichen Interesses an der Strafverfolgung ein Einschreiten von Amts wegen für geboten hält.

\section{b) Verfahrensrecht}

Neben der Schaffung einer eigenständigen Strafnorm verfolgt der Gesetzesentwurf eine Ausweitung des Rechts der Untersuchungshaft. Gemäß Art. 2 Nr. 1 des Gesetzesentwurfs soll $\S 238$ Abs. 2 bis 4 StGB EF in $\S 112$ a Abs. 1 Nr. 1 StPO aufgenommen werden, um mittels einer sog. Deeskalationshaft eine Handhabe gegen besonders gefährliche Täter zu schaffen. ${ }^{52}$ Nach der Entwurfsbegründung könne dem Opfer in gravierenden Fällen des Stalking oftmals nur dann geholfen werden, wenn der durch den Täter in Gang gesetzte Terror durch dessen Inhaftierung unterbrochen werde, wozu die zeitlich begrenzte Ingewahrsamnahme nach Polizeirecht keine effektive Handhabe biete. $^{53}$

In verfahrensrechtlicher Hinsicht ist zudem darauf hinzuweisen, dass der Grundtatbestand des $§ 238$ StGB EF gemäß Art. 2 Nr. 2 des Gesetzesentwurfs als Privatklagedelikt ausgestaltet werden soll. Insofern ist vorgesehen, den Straftatbestand des $§ 238$ Abs. 1 StGB EF als Nr. 4a in $\S 374$ Abs. 1 StPO aufzunehmen. Zudem soll $§ 238$ StGB EF zur Ermöglichung einer Nebenklage in den Katalog des $§ 395$ StPO einbezogen werden.

\section{Stellungnahme}

Der vom Bundesrat vorgelegte Gesetzesentwurf erweist sich in mehrfacher Hinsicht als problematisch.

52 Gesetzesentwurf des Bundesrats, BT-Drs. 15/5410, S. 6.

53 Vgl. Gesetzesentwurf des Bundesrats, BT-Drs. 15/5410, S. 7. 


\section{a) Bestimmtheit}

Der Gesetzesentwurf krankt vor allem daran, dass der neue Straftatbestand des $§ 238$ StGB EF in hohem Maße unbestimmt ist.

Dies gilt zum einen für die Aufnahme der »ebenso schwerwiegenden Handlungen« in den Tatbestand des $§ 238$ Abs. 1 Nr. 3 StGB EF. Grundsätzlich ist zwar auch innerhalb des Strafrechts die Verwendung unbestimmter Rechtsbegriffe zulässig. Gerade die Vielschichtigkeit möglicher Stalking-Handlungen erfordert offene Formulierungen, um den Tätern die Möglichkeit der Umgehung des Tatbestandes von vornherein abzuschneiden. ${ }^{54}$ Die Verwendung unbestimmter Rechtsbegriffe ist jedoch nur zulässig, wenn diese unter Berücksichtigung des Normzwecks zumindest konkretisierbar sind. Die Begründung des dem Gesetzesentwurf zugrunde liegenden Antrags des Lands Hessen geht davon aus, dass sich die inhaltliche Bestimmtheit aus dem Zusammenhang mit den anderen genau umschriebenen Begehungsalternativen sowie der zur Vollendung des Delikts erforderlichen Wirkung auf Seiten des Opfers ergäbe. ${ }^{55} \mathrm{Be}-$ trachtet man jedoch die Beispiele, die sich der Gesetzesbegründung für den Anwendungsbereich von $\S 238$ Abs. 1 Nr. 3 StGB EF entnehmen lassen (z. B. unrichtige Anzeigen in Zeitungen, das Hinterlassen von Mitteilungen, die Verächtlichmachung des Opfers bei Freunden und Kollegen, die Überwachung des Freundes- und Bekanntenkreises des Opfers, die Bestellung von Waren oder das Abonnieren von Zeitschriften unter dem Namen des Opfers), ${ }^{56}$ bestehen insoweit erhebliche Zweifel. Die im Zusammenhang mit den »ebenso schwerwiegenden Handlungen« umschriebenen Begehungsalternativen sind stark heterogen und erlauben kaum Rückschlüsse darauf, was als »ebenso schwerwiegende Handlung « betrachtet werden könnte. Dieser Erkenntnis verschließt sich auch die Begründung des Gesetzesentwurfs nicht, in der deutlich zum Ausdruck gebracht wird, dass durch $\S 238$ Abs. 1 Nr. 3 StGB EF dem Umstand Rechnung getragen werden soll, dass sich der durch einen Stalker vollführte Terror einer abschließenden gesetzlichen Eingrenzung entzieht. ${ }^{57}$ Leider scheint diese Einsicht bei der Formulierung des Gesetzestextes wieder aus den Augen verloren gegangen zu sein. ${ }^{58} \mathrm{Da}$ auch die Formulierung des tatbestandlichen Erfolgs sehr weit und indifferent gefasst ist, erscheint auch diese wenig tauglich, die »ebenso schwerwiegende Handlung « zu konkretisieren. ${ }^{59}$

54 Wagner, in: Plenarprotokoll des Bundesrats, Stenografischer Bericht über die 809. Sitzung, S. 86 C; ders., in: Plenarprotokoll des Bundestags, Stenografischer Bericht über die 178. Sitzung, S. 16781 B, C.

55 Gesetzesantrag des Landes Hessen, BR-Drs. 551/04, S. 10 des Gesetzesentwurfs. Vgl. die Erklärung des hessischen Staatsministers Wagner, in: Plenarprotokoll des Bundesrats, Stenografischer Bericht über die 802. Sitzung, Anlage 30, S. 399 D. Vgl. auch Meyer, ZStW 115 (2003), 249, 288.

56 Gesetzesentwurf des Bundesrats, BT-Drs. 15/5410, S. 7.

57 Gesetzesentwurf des Bundesrats, BT-Drs. 15/5410, S. 7.

58 Vgl. Hartenbach, Plenarprotokoll des Bundestags, Stenografischer Bericht über die 178. Sitzung, S. 16786 A.

59 A. A. Wagner, in: Plenarprotokoll des Bundesrats, Stenografischer Bericht über die 809. Sitzung, S. 86 C. 
Eine parallele Problematik besteht hinsichtlich des Tatbestandsmerkmals der »Eignung für eine erhebliche Beeinträchtigung «. Die Ausgestaltung des Tatbestands als Eignungsdelikt soll dazu beitragen, dass nur solche Fälle erfasst werden, in denen die Tat bei einer Beurteilung ex ante die Gefahr in sich trägt, dass das Opfer auf Grund des vom Täter entfalteten Terrors in wesentlichen Belangen nicht mehr so leben kann wie zuvor. ${ }^{60}$ Die Frage, wann ein Verhalten des Täters insofern »geeignet « erscheint, lässt sich kaum allgemein beantworten. Die Eignung knüpft im Gegenteil im Wesentlichen an die subjektive Sicht des (vermeintlichen) Stalking-Opfers an. Was der eine als unzumutbare Nachstellung oder Verfolgung empfindet, erscheint dem anderen als unerhebliche Belästigung. Die notwendige Abgrenzung zwischen der bloßen Belästigung und der rechtlich erheblichen Beeinträchtigung anhand des Kriteriums der Eignung erscheint zweifelhaft. Dies gilt umso mehr, wenn - wie in der Gesetzesbegründung ${ }^{61}$ - der Eindruck erweckt wird, dass die Eignung durch Bezug auf »das Opfer « konkret zu ermitteln ist. ${ }^{62}$ Selbst wenn hier auf die Sicht eines objektiven Dritten abgestellt werden sollte, ist fraglich, wo die objektivierte Grenzziehung zwischen Belästigung und Beeinträchtigung verlaufen sollte. Subjektive Empfindungen des Opfers sollten nicht Tatbestandsmerkmale einer Norm sein, da sie kaum standardisierbar sind und daher keinen verlässlichen Maßstab für die Bestimmung strafbaren Verhaltens bilden. ${ }^{63}$ Die Aufnahme derartiger Merkmale in den Tatbestand führt darüber hinaus zu erheblichen Nachweisproblemen. ${ }^{64}$

Schließlich ist auch das Verhältnis einiger weiterer unbestimmter Rechtsbegriffe zueinander nicht eindeutig. Insoweit erscheint ein Verstoß gegen das Bestimmtheitsgebot auch vor dem Hintergrund gegeben, dass die unterschiedliche Bedeutung der kumulativen Tatbestandsvoraussetzungen »nachhaltig «, »fortgesetzt « und »erheblich« nicht hinreichend erkennbar ist. ${ }^{65}$ So ist zum einen nicht vorstellbar, wie eine Handlung, die geeignet ist, eine erhebliche Beeinträchtigung herbeizuführen, nicht zugleich gravierend und damit nachhaltig sein sollte; zum anderen dürfte eine Belästigung, wenn sie schon gravierend und damit nachhaltig ist, stets die Eignung für eine erhebliche Lebensbeeinträchtigung besitzen. ${ }^{66}$ In Bezug auf das Merkmal »fortgesetzt « ist zudem zu kritisieren, dass nach dem Verständnis der Gesetzesbegründung das Vorliegen dieses Merkmals erst nach fünfmaligen Stalking-Handlungen gegeben sein soll. ${ }^{67}$ In seiner Stellungnahme zum Gesetzesentwurf weist der Deutsche Richterbund mit Recht darauf hin, dass diese Auslegung des gleichsam in $§ 335$ Abs. 2 Nr. 2 StGB ver-

60 Vgl. Gesetzesentwurf des Bundesrats, BT-Drs. 15/5410, S. 7.

61 Vgl. Gesetzesentwurf des Bundesrats, BT-Drs. 15/5410, S. 7, der insoweit auf das konkrete Opfer (»das Opfer«) abstellt.

62 Stellungnahme des Deutschen Richterbundes, Nr. 2. Die Stellungnahme ist abrufbar unter: http://www.drb.de/pages/html/stellung/st-stalking.html

63 Vgl. Meyer, ZStW 115 (2003), 249, 283; a. A. von Pechstaedt [Fn. 3], S. 155.

64 Meyer, ZStW 115 (2003), 249, 283. Aus ähnlichen Erwägungen lehnte die damalige Justizministerin Däubler-Gmelin in einem Zeitungsinterview (zitiert bei Pollähne, NK 2002, 56, 58; Kerbein/Pröbsting, ZRP 2002, 76, 78) die Schaffung eines gesonderten Straftatbestandes für das Stalking ab.

65 Stellungnahme des Deutschen Richterbundes, Nr. 1.

66 Vgl. Stellungnahme des Deutschen Richterbundes, Nr. 1.

67 Gesetzesentwurf des Bundesrats, BT-Drs. 15/5410, S. 7. 
wendeten Begriffs weder mit der einschlägigen BGH-Rechtsprechung noch mit der abweichenden in der Literatur vertretenen Auffassung zu vereinbaren ist. ${ }^{68}$ Der Einheit des Strafrechts dürfte insoweit kaum ein Dienst erwiesen werden.

Der Tatbestand enthält damit gleich mehrere unbestimmte Rechtsbegriffe, deren Verhältnis zueinander zudem nicht geklärt ist, so dass in der Summe ein hohes Maß an Unbestimmtheit zu verzeichnen ist. ${ }^{69}$ Der Strafvorschrift kann vor diesem Hintergrund nicht entnommen werden, was genau strafbar ist und was nicht. ${ }^{70}$ Dies widerspricht jedoch dem verfassungsrechtlich vorgeschriebenen Bestimmtheitsgebot des Art. 103 Abs. 2 GG. $^{71}$

\section{b) Qualifikationstatbestände}

Fraglich ist zudem, ob die Qualifikationstatbestände des $§ 238$ Abs. 2 bis 4 StGB EF mit ihren zum Teil erheblichen Strafandrohungen angemessen und erforderlich sind. ${ }^{72}$ In diesem Rahmen ist zu bedenken, dass es sich bei den Tatbeständen gemäß $\S 238$ Abs. 3 und 4 StGB EF um Verbrechen handelt. Vor diesem Hintergrund hat der Deutsche Richterbund vorgeschlagen, anstellte der Absätze 2 bis 4 einen Absatz mit einer Regelung für besonders schwere Fälle bei einem Strafrahmen von 3 Monaten bis zu 10 Jahren vorzusehen und als Regelbeispiele eine Zusammenfassung der bisherigen Tatfolgen in den Absätzen 2 bis 4 aufzunehmen. ${ }^{73}$ Dies böte insbesondere den Vorteil einer deutlich erhöhten Flexibilität bei der Strafzumessung. Da die in den betreffenden Vorschriften aufgeführten Normen in der bisherigen hessischen Entwurfsfassung gar nicht vorgesehen waren, liegt es zudem nahe, dass ein entsprechender Regelungsbedarf aktuell nicht vorliegt. ${ }^{74}$ Schließlich ist $\mathrm{zu}$ berücksichtigen, dass in Fällen von schwerem Stalking regelmäßig neben einem gegebenenfalls bestehenden Stalking-Straftatbestand weitere Straftatbestände einschlägig sind (etwa bei Körperverletzungs-, Sexual- oder Tötungsdelikten), die eine ausreichende Gewähr für eine angemessene Sanktionierung auch schwerer Stalking-Fälle sicherstellen. ${ }^{75}$

68 Vgl. Stellungnahme des Deutschen Richterbundes, Nr. 3 lit. a, b. Der BGH nimmt eine fortgesetzte Tatbegehung bei entsprechendem Fortsetzungswillen auch bei nur einer Handlung an, während die Literatur drei Handlungen verlangt.

69 Vgl. Schewe-Gerigk, in: Plenarprotokoll des Bundestags, Stenografischer Bericht über die 178. Sitzung, S. 16784 B.

70 So auch die Auffassung von Mertin, in: Plenarprotokoll des Bundesrats, Stenografischer Bericht über die 809. Sitzung, S. 87 A.

71 Stellungnahme der Bundesregierung, BT-Drs. 15/5410, S. 9; so auch der in einer Pressemeldung des rheinland-pfälzischen Justizministeriums vom 21.07.2004 geäußerte Standpunkt; vgl. Bachmaier, in: Plenarprotokoll des Bundestags, Stenografischer Bericht über die 178. Sitzung, S. 16782 C.

72 So die Stellungnahme des Bundesrats, BR-Drs. 617/05, S. 1; vgl. auch Wagner, in: Plenarprotokoll des Bundesrats, Stenografischer Bericht über die 814. Sitzung, S. 326 D.

73 Vgl. Stellungnahme des Deutschen Richterbundes, Nr. 4. Dieser Vorschlag kommt dem ursprünglichen Gesetzesantrag des Landes Hessen nahe. Vgl. hierzu Gesetzesantrag des Landes Hessen, BR-Drs. 551/04.

74 Stellungnahme des Deutschen Richterbundes, Nr. 4.

75 Dies verkennt Wagner, in: Plenarprotokoll des Bundesrats, Stenografischer Bericht über die 814. Sitzung, S. 326 D. 


\section{c) Deeskalationshaft}

In prozessualer Hinsicht besonders bedenklich erscheint die geplante Ausweitung des Haftgrundes der Wiederholungsgefahr ( $1112 \mathrm{a}$ StPO) durch die im Entwurf vorgesehene Deeskalationshaft. Auch wenn die Möglichkeit der Inhaftierung des Täters in gravierenden Fällen einen wichtigen Beitrag zur Deeskalation und damit zum wirksamen Opferschutz leisten könnte ${ }^{76}$ sind die verfassungsrechtlichen Anforderungen an dieses einschneidende strafprozessuale Mittel nicht aus den Augen zu verlieren.

Das Bundesverfassungsgericht verlangt für die Verhängung von Untersuchungshaft eine schwerwiegende Beeinträchtigung der Rechtsordnung. ${ }^{77}$ Bei Straftaten gemäß $\S 238$ Abs. 2 bis 4 StGB EF kann jedoch zum einen nicht generell-abstrakt von einer schwerwiegenden Beeinträchtigung der Rechtsordnung ausgegangen werden, zum anderen kann nicht abstrakt-generell von der Gefahr ausgegangen werden, der Täter werde weitere erhebliche Straftaten gleicher Art begehen. ${ }^{78}$ Die vorgeschlagene Ausweitung der Anlasstaten gemäß § 112a StPO genügt damit nicht den verfassungsrechtlichen Anforderungen, die an den Haftbefehl der Wiederholungsgefahr zu stellen sind. ${ }^{79}$ Insbesondere $§ 238$ Abs. 2 StGB EF weist nicht die Schwere auf, die für Anlasstaten nach § 112a StPO als verfassungsrechtlich unbedenklich angesehen werden kann. ${ }^{80}$ Daneben ergeben sich sogar aus der Begründung des Gesetzesentwurfs selbst erhebliche Bedenken in Bezug auf die geplante Deeskalationshaft. Obwohl die Gesetzesbegründung deutlich zum Ausdruck bringt, dass es sich bei Stalkern zumeist um ansonsten strafrechtlich noch nicht in Erscheinung getretene Personen in geordneten sozialen Verhältnissen handelt ${ }^{81}$ scheint diese Erkenntnis bei der geplanten Erweiterung der Haftgründe nicht berücksichtigt worden zu sein. ${ }^{82}$

Von diesen rechtlichen Bedenken abgesehen ist auch der praktische Nutzen einer Deeskalationshaft zumindest in Frage zu stellen. Da eine Untersuchungshaft nicht unbegrenzt und dauerhaft bestehen bleiben kann (vgl. §§ 121 Abs. 1, 122a StPO), wird

76 Vgl. Wagner, in: Plenarprotokoll des Bundestags, Stenografischer Bericht über die 178. Sitzung, S. $16781 \mathrm{C}$.

77 BVerfGE 35, 185, 192 = NJW 1973, 1363, 1365.

78 Stellungnahme der Bundesregierung, BT-Drs. 15/5410, S. 9; a. A. Merk, in: Plenarprotokoll des Bundesrats, Stenografischer Bericht über die 809. Sitzung, Anlage 10, S. 113 A.

79 Stellungnahme der Bundesregierung, BT-Drs. 15/5410, S. 9. Auch das Land SchleswigHolstein hatte bereits vor der Beschlussfassung des Gesetzesentwurfs durch den Bundesrat die Ausweitung des Katalogs der Anlasstaten in Gestalt der Eskalationsgefahr aus »grundsätzlichen Bedenken« abgelehnt. Vgl. hierzu den Antrag des Landes Schleswig-Holstein, BR-Drs. 551/2/04, S. 11.

80 Stellungnahme der Bundesregierung, BT-Drs. 15/5410, S. 9.

81 Gesetzesentwurf des Bundesrats, BT-Drs. 15/5410, S. 7.

82 Vgl. Hartenbach, in: Plenarprotokoll des Bundestags, Stenografischer Bericht über die 178. Sitzung, S. 16786 B. 
vielfach mit einer eher kurzfristigen Inhaftierung zu rechnen sein, die im Ergebnis oftmals nur noch zu weiterer Gewalt führen dürfte. ${ }^{83}$

\section{Entwurf der Bundesregierung}

Die Bundesregierung legte am 10. August 2005 gleichsam einen Gesetzesentwurf vor.

\section{Inhalt}

Der Entwurf unternimmt vor dem Hintergrund bereits geäußerter Bedenken hinsichtlich der Bestimmtheit des Gesetzesentwurfs des Bundesrats den Versuch der Formulierung eines hinreichend bestimmten Tatbestandes und verzichtet auf die Ausweitung des Haftgrundes der Wiederholungsgefahr.

\section{$\S 241 b$ StGB EF}

Nachstellung

(1) Wer einem Menschen unbefugt nachstellt, indem er beharrlich

1. seine räumliche Nähe sucht,

2. unter Verwendung von Telekommunikationsmitteln oder sonstigen Mitteln der Kommunikation oder über Dritte Kontakt zu ihm herzustellen versucht,

3. unter missbräuchlicher Verwendung von dessen personenbezogenen Daten Bestellungen von Waren oder Dienstleistungen für ihn aufgibt oder Dritte veranlasst, mit diesem Kontakt aufzunehmen, oder

4. ihn mit der Verletzung von Leben, körperlicher Unversehrtheit, Gesundheit oder Freiheit seiner selbst oder einer ihm nahestehenden Person bedroht, und dadurch seine Lebensgestaltung schwerwiegend und unzumutbar beeinträchtigt, wird mit Freiheitsstrafe bis zu drei Jahren oder mit Geldstrafe bestraft.

(2) Die Tat nach Absatz 1 wird nur auf Antrag verfolgt, es sei denn, dass die Strafverfolgung wegen des besonderen öffentlichen Interesses an der Strafverfolgung ein Einschreiten von Amts wegen für geboten hält.

In Parallele zum Entwurf des Bundesrats sieht der Entwurf der Bundesregierung in prozessualer Hinsicht gleichsam die Ausgestaltung des Straftatbestandes als Antragssowie Privatklagedelikt vor.

\section{Stellungnahme}

Auch der Entwurf der Bundesregierung ist zu kritisieren.

83 Schewe-Gerigk, in: Plenarprotokoll des Bundestags, Stenografischer Bericht über die 178. Sitzung, S. 16784 C. 


\section{a) Effektivität}

Der Gesetzesentwurf der Bundesregierung vermeidet eine offene Formulierung des Tatbestandes durch die Verwendung eines abschließenden Katalogs bestimmter Stalking-Handlungen. Durch den Verzicht auf eine generalklauselartige Umschreibung des Tatbestandes erweist sich der Vorschlag der Bundesregierung unter Bestimmtheitsgesichtspunkten deutlich weniger problematisch. Die Effektivität der Vorschrift ist hierdurch jedoch in Frage gestellt, da die Norm ausschließlich die genannten Handlungen unter Strafe stellt. ${ }^{84}$ Dieser abschließende Katalog ist in Anbetracht der Vielschichtigkeit und Heterogenität von Stalking-Verhaltensweisen geradezu eine Einladung an die Täter, durch Umgehungsstrategien einer Strafbarkeit zu entgehen und auf andere Weise, Kontakt zu dem Opfer zu suchen.

\section{b) Bestimmtheit}

Davon abgesehen erscheint der Entwurf trotz seiner gegenteiligen Zielsetzung im Hinblick auf die verfassungsrechtlichen Bestimmtheitsanforderungen problematisch. Fraglich ist vor allem, wann eine »schwerwiegende und unzumutbare Beeinträchtigung der Lebensführung « vorliegen soll. Ein Blick in die Gesetzesbegründung bringt hervor, dass nur erhebliche, nach Außen sich manifestierende Beeinträchtigungen ausschlaggebend sein sollen. Insoweit werden die Beschaffung einer Geheimnummer oder der Wechsel des Wohnsitzes genannt. Diese Kriterien sind jedoch kaum geeignet, einen allgemeingültigen Maßstab zu liefern, da die Beeinträchtigung der Lebensführung maßgeblich auf der Empfindsamkeit des Opfers beruht. Was den einen zum Wechsel des Wohnsitzes veranlasst, lässt den anderen noch kalt. Wo hier die objektivierbare Grenzziehung zwischen hinnehmbaren Belästigungen und unzumutbaren Beeinträchtigungen liegen soll, ist fraglich. Insofern erscheint auch der Gesetzesentwurf der Bundesregierung wenig geeignet, als neuer Straftatbestand gegen Stalking zu firmieren.

\section{c) Kausalität}

Schließlich erweist sich der Gesetzesentwurf der Bundesregierung auch im Hinblick auf die Kausalität des Tatbestandes als problematisch. Dieser soll nach dem Vorschlag der Bundesregierung im Unterschied zum Gesetzesentwurf des Bundesrats nicht als Eignungs-, sondern als konkretes Erfolgsdelikt ausgestaltet werden, so dass es eines Kausalitätsnachweises zwischen Täterverhalten und tatbestandlichem Erfolg bedarf. ${ }^{85}$ Da in vielen Stalking-Fällen bereits der Nachweis der Stalking-Handlungen selbst erhebliche Schwierigkeiten bereitet (vielfach finden Kontaktaufnahmeversuche allein zwischen dem Stalker und seinem Opfer statt, so dass insbesondere auf den Zeugenbeweis regelmäßig nicht zurückgegriffen werden kann), wird deutlich, dass ein darüber

84 Vgl. Stellungnahme des Bundesrats, BR-Drs. 617/05, S. 1, mit dem Hinweis auf erhebliche Strafbarkeitslücken.

85 Vgl. Stellungnahme des Bundesrats, BR-Drs. 617/05, S. 1; vgl. auch Wagner, in: Plenarprotokoll des Bundesrats, Stenografischer Bericht über die 814. Sitzung, S. 326D. 
hinausgehender Nachweis in Bezug auf den durch die jeweilige Handlung konkret hervorgerufenen Erfolg mit ungleich höheren Beweisschwierigkeiten belastet ist.

\section{Schaffung eines speziellen Stalking-Straftatbestandes}

Es ist anzuerkennen, dass Stalking eine Verhaltensweise ist, welche eine Reihe von Handlungen vereinigen kann, wovon jede für sich nicht als ernst erscheinen kann, aber wenn sie über eine bestimmte Zeit verbunden sind, auf das Opfer eine ernste Einwirkung haben kann, die fortwährende Belästigung, Bedrängnis, Angst, Unbehagen und oft ernstere Beeinträchtigungen des psychischen Wohlseins des Opfers verursacht. ${ }^{86}$ Allerdings darf nicht jede bloße Belästigung mit strafrechtlichen Mitteln sanktioniert werden.

Vor diesem Hintergrund ist fraglich, ob die Einführung eines besonderen Straftatbestandes zur Sanktionierung von Stalking geboten ist, um einen adäquaten Schutz vor Stalkern gewährleisten zu können.

\section{Für und Wider}

Für eine selbständige Deliktsumschreibung spricht, dass in den vorhandenen Straftatbeständen nicht der Kern des Unrechts ausgedrückt wird, der im systematischen Behelligen des Opfers liegt. ${ }^{87}$ Die Bestimmungen des Strafgesetzbuchs sind auf einzelne Ergebnisse zugeschnitten, wohingegen das Wesen der unzumutbaren Belästigung in einer fortwährenden Beeinträchtigung liegt. ${ }^{88}$ Insoweit ist jedoch zu beachten, dass das bloße »Fehlen« eines Straftatbestandes schwerlich als Argument für dessen Schaffung angeführt werden kann. Zudem besteht jedenfalls in Gestalt des GewSchG eine mittelbare Strafbarkeit des Stalking, ${ }^{89}$ so dass der Verhaltensunwert des Phänomens durchaus bereits unter der geltenden Gesetzeslage erfasst werden kann. In diesem Zusammenhang ist zudem darauf hinzuweisen, dass in der Schaffung eines neuen, eigenständigen Stalking-Straftatbestandes die Gefahr liegt, dass sich verschiedene Straftatbestände überlagern und die Konturen der bisher existierenden Tatbestände verschwimmen. ${ }^{90}$

Die Befürworter eines Stalking-Straftatbestandes stellen sich regelmäßig auf den Standpunkt, dass der aktuelle strafrechtliche Schutzbereich unzureichend sei, da viele Stalking relevante Handlungen mit den gängigen strafrechtlichen Mitteln nicht sanktioniert werden könnten. ${ }^{91}$ Es ist jedoch zu berücksichtigen, dass das Strafrecht als $u l$ tima ratio nicht jede Belästigung oder unangenehme Kontaktaufnahme unterbinden kann, sondern nur bei wesentlichen Einbußen im Sinne einer Verletzung von Rechts-

86 von Pechstaedt [Fn. 3], S. 132.

87 von Pechstaedt [Fn. 3], S. 141.

88 Erklärung des hessischen Staatsministers Wagner, in: Plenarprotokoll des Bundesrats, Stenografischer Bericht über die 802. Sitzung, Anlage 30, S. 399 C.

89 Hierzu: B I 2 b.

90 A. A.: von Pechstaedt [Fn. 3], S. 154 f.

91 Vgl. Kerbein/Pröbsting, ZRP 2002, 76, 78. 
gütern oder gravierenden Gefährdungen zur Anwendung kommen sollte. ${ }^{92}$ Ernsthafte Belästigungen werden vielfach bereits durch bestehende Strafvorschriften erfasst. ${ }^{93}$ Gerade vor diesem Hintergrund erscheint die Notwendigkeit der Schaffung eines Sondertatbestandes keinesfalls zwingend. Dies gilt auch und vor allem mit Blick auf die einschlägigen Regelungen des Gewaltschutzgesetzes, die eine Möglichkeit bieten, gegen Stalker vorzugehen. An dieser Stelle soll jedoch nicht verschwiegen werden, dass sich auch das GewSchG wegen der engen Voraussetzungen, die an den Erlass einer einstweiligen Verfügung gestellt werden, sowie der geringen Strafandrohung nicht als gänzlich unproblematisch darstellt. Diese Schwächen sollten allerdings nicht durch einen neuen Straftatbestand, sondern durch eine Anpassung des Gewaltschutzgesetzes, namentlich durch Ausweitung des Katalogs in $\S 1$ Abs. 2 S. 1 lit. b GewSchG sowie eine Erhöhung des Strafrahmens gemäß $§ 4$ GewSchG kompensiert werden. In diese Richtung tendierte bereits ein Gesetzesvorschlag des Landes Rheinland-Pfalz, ${ }^{94}$ der leider nicht weiter verfolgt wurde, obwohl er durchaus als taugliche Orientierungshilfe für etwaige gesetzgeberische Maßnahmen hätte herangezogen werden können.

Für die Schaffung eines eigenen Stalking-Straftatbestandes werden auch praktische Gesichtspunkte angeführt. So wird hervorgehoben, dass eine Transformation ins Strafgesetzbuch den großen Vorteil habe, dass das gestalkte Opfer sofort polizeiliche Hilfe in Anspruch nehmen könnte und sich die Polizei nicht mehr darauf berufen könne, dass »noch nichts passiert « sei. ${ }^{95}$ Die Festlegung eines allgemeinen Stalking-Straftatbestandes hätte zur Folge, dass das gesamte Verfahren von Amts wegen geführt und dadurch das Opfer - im Gegensatz zum GewSchG, wonach eine Strafbarkeit erst nach Titulierung eines zu substantiierenden Unterlassungsanspruchs in Frage kommt - entlastet wird. ${ }^{96}$ Allerdings ist zu beachten, dass auch die Einleitung eines Straf- bzw. Ermittlungsverfahrens regelmäßig von einer Strafanzeige, also einem Tätigwerden des Opfers abhängt. ${ }^{97}$ In diesem Zusammenhang ist darauf hinzuweisen, dass die aktuellen Gesetzesentwürfe eine Ausgestaltung des Stalking-Straftatbestandes als Antrags- und Privatklagedelikt vorsehen. Zudem wird das Opfer für den Fall einer Hauptverhandlung gegen den Täter vor Gericht aussagen müssen - eine Situation, die im Vergleich zum Zivilprozess, bei dem eine Vertretung durch den Rechtsbeistand möglich ist, eine erhebliche Belastung des Opfers darstellt.

Gegen die Schaffung eines eigenständigen Sonderstraftatbestandes sprechen vor allem gesetzestechnische Erwägungen. Es ergeben sich vor allem dadurch Schwierig-

92 Vgl. Gesetzesantrag des Landes Hessen, BR-Drs. 551/04, S. 6 des Gesetzesentwurfs; vgl. auch Pollähne, NK 2002, 56, 58; von Pechstaedt [Fn. 3], S. 139.

93 Hierzu: B I 1.

94 Der Gesetzesentwurf des rheinland-pfälzischen Justizministeriums kann auf den InternetSeiten des Ministeriums unter der Rubrik »Presse« abgerufen werden (Pressemitteilung vom 21.07.2004). Vgl. http://www.justiz.rlp.de.

95 Kerbein/Pröbsting, ZRP 2002, 76, 78.

96 Kerbein/Pröbsting, ZRP 2002, 76, 78; vgl. auch Antrag des Landes Schleswig-Holstein, BR-Drs. 551/2/04, S. 5.

97 Borchert, FPR 2004, 239, 240; vgl. auch die Pressemeldung des rheinland-pfälzischen Justizministeriums vom 21.07.2004. 
keiten, dass eine Unrechtstypisierung der von Stalkern begangenen Belästigungen angesichts der heterogenen Fallgestaltungen nur schwer gelingt und einige Zweifel hinsichtlich der Bestimmtheitsanforderungen des Art. 103 Abs. 2 GG aufwirft. ${ }^{98}$ Nach deutschem Rechtsverständnis lässt sich unter Berücksichtigung der verfassungsrechtlich gebotenen Bestimmtheitsanforderungen das komplexe und facettenreiche Phänomen Stalking nicht sauber in einem Straftatbestand erfassen. ${ }^{99}$ Zudem erscheint die Sanktionierung eines Verhaltens, das insbesondere von subjektiv völlig unterschiedlich ausgeprägten Empfindsamkeiten abhängt, schwerlich realisierbar. ${ }^{100}$ In diesem Zusammenhang ist zu erwähnen, dass mit zunehmender Bekanntheit des Stalking auch das Phänomen der »falschen Opfer « verstärkt in Erscheinung tritt. ${ }^{101}$ Neuere Studien kommen zu dem Ergebnis, dass in etwa zehn bis 20\% aller Fälle Personen fälschlicherweise angeben, von Stalkern belästigt zu werden. ${ }^{102}$

Abschließend ist zu konstatieren, dass die Probleme, die zu tatsächlichen Schutzlücken im Bereich Stalking führen, weniger im Rechtlichen als vielmehr im Faktischen $\mathrm{zu}$ liegen scheinen. ${ }^{103}$ So fehlt es insbesondere am Wissen der zuständigen Entscheidungsträger über Relevanz und Phänomen des Stalking, was zur Folge hat, dass von den vorhandenen rechtlichen Möglichkeiten nur unzureichend Gebrauch gemacht wird. ${ }^{104}$

\section{Alternativer Ansatz}

Alternativ zur Verschärfung und Ausweitung des Strafrechts erscheint eine konsequente Anwendung bestehender Vorschriften als probates Mittel. ${ }^{105}$ Eine effektive Anwendung bestehender Möglichkeiten setzt jedoch voraus, dass die betroffenen Behörden, insbesondere die Polizei, mit dem Problem des Stalking vertraut gemacht werden. Zu diesem Zweck erscheint es sinnvoll, Expertengruppen einzurichten, die sich speziell dieser Thematik widmen. ${ }^{106}$ In den USA sind derartige Spezialgruppen längst prak-

98 Dieses Problem wird auch im Rahmen des Gesetzesantrages des Landes Hessen, BRDrs. 551/04, S. 5 f. des Gesetzesentwurfs, gesehen. Vgl. zu den Problemen des Gewaltbegriffs (auch im Zusammenhang mit Stalking): Kube, Kriminalistik 1999, $161 \mathrm{ff}$.

99 Borchert, FPR 2004, 239, 240; a. A. von Pechstaedt [Fn. 3], S. 155, der insbesondere für subjektive Aspekte des Tatbestandes auf »objektive Kriterien, so die Ansicht eines außenstehenden (vernünftigen) Beobachters « zurückgreifen möchte.

100 Borchert, FPR 2004, 239, 240

101 Hoffmann, Kriminalistik 2003, 726, 730; vgl. auch von Pechstaedt [Fn. 3], S. 47.

102 Hoffmann, Kriminalistik 2003, 726, 730.

103 Meyer, ZStW 115 (2003), 249, 291.

104 Meyer, ZStW 115 (2003), 249, 291.

105 So auch der Standpunkt der Bundesregierung in der Stellungnahme zum Gesetzesentwurf, BT-Drs. 15/5410, S. 9.

106 In Deutschland ist insoweit das Anfang 2001 gestartete Stalking-Projekt der Polizei Bremen hervorzuheben. Hierzu eingehend: Oehmke, in: Bettermann/Feenders [Fn. 3], S. 210 ff. Kritisch zur Effektivität des Projekts: Bachmaier, in: Plenarprotokoll des Bundestags, Stenografischer Bericht über die 178. Sitzung, S. 16782 B, C. Hinzuweisen ist auch auf ein Hamburger Modellprojekt zur Implementierung eines Kompetenzzentrums. Dazu: Seifert et al., in: Bettermann/Feenders [Fn. 3], S. 235 ff. 
tische Wirklichkeit. Bereits im Jahre 1990 gründete das Los Angeles Police Department als erste Polizeibehörde weltweit eine Spezialeinheit für derartige Fälle. ${ }^{107}$ Einen ersten wichtigen Beitrag könnten insbesondere die Einrichtung von spezialisierten Referaten bei den Staatsanwaltschaften sowie die verstärkte Verfolgung und Aburteilung von Stalking-Taten im beschleunigten Verfahren leisten. ${ }^{108}$

Ein effektiver, wirklich opferschützender Umgang mit Stalking ist nur durch die Entwicklung eines Gesamtkonzepts möglich, das eine Vielzahl von unterschiedlichen Instrumenten miteinander verbindet und als dessen Bestandteil vor allem Beratungsund Therapiemöglichkeiten für Opfer und Täter bietet. ${ }^{109}$ Insoweit ist ein ganzes Maßnahmebündel erforderlich, das straf-, polizei- und zivilrechtliche sowie soziale, außerrechtliche Instrumente ${ }^{110}$ umfasst. $^{111}$

\section{E. Fazit und Ausblick}

Das Phänomen Stalking ist ein ernst zu nehmendes Problem. Durch das GewSchG hat der Gesetzgeber jedoch bereits eine richtige Weichenstellung zum Schutz der Opfer vorgenommen. In Gestalt der strafrechtlich sanktionierbaren Unterlassungsverfügung steht einem Stalking-Opfer ein gangbarer Weg zur Beendigung von Stalking-Handlungen zur Verfügung. Zudem eröffnet das zivilgerichtliche Verfahren die Möglichkeit, eine Beruhigung der Gemüter herbeizuführen, so dass der Streit durch eine Einigung im Vergleichswege beigelegt werden kann. ${ }^{12}$

Gegen die Einführung eines Sondertatbestandes sprechen vor allem die Schwierigkeiten der Erfassung des strafrechtlich relevanten Verhaltens durch eine Norm. Das Strafrecht gerät daher im Bereich des Stalking an die Grenzen dessen, was sich in seinem Rahmen sinnvoll regeln lässt. ${ }^{113}$ Ein Straftatbestand, der alle möglichen Tathandlungen in einer abstrakten Formulierung erfassen möchte, verliert seine Konturen. ${ }^{114}$ Auch die

107 Vgl. Hoffmann, Kriminalistik 2001, 34, 36; zum niederländischen Modell des AWARE-Projekts vgl. von Pechstaedt [Fn. 3], S. 13.

$108 \mathrm{Vgl}$. Hartenbach, in: Plenarprotokoll des Bundesrats, Stenografischer Bericht über die 809. Sitzung, S. 88 C.

109 Meyer, ZStW 115 (2003), 249, 293.

110 Berichte über Selbsthilfe bieten: Tholen, in: Bettermann/Feenders [Fn. 2], S. 209 ff.; Pilath, in: Bettermann/Feenders [Fn. 3], S. 219 ff. Vgl. auch die Websites http://www.stalkingforum.de und http://www.liebeswahn.de.

111 Vgl. Hoffmann, Kriminalistik 2001, 34 ff; Kube, Kriminalistik 1999, 161, 165; Meyer, ZStW 115(2003), 249, 293; von Pechstaedt, in: Bettermann/Feenders [Fn. 3], S. 147, 167; vgl. auch Pelikan, Forschungsbericht Psychoterror, 2003, S. 113 ff. Diesem Standpunkt hat sich auch die Bundesregierung in ihrer Stellungnahme zum Gesetzesentwurf angeschlossen. Vgl. BT-Drs. 15/5410, S. 9; vgl. auch Hartenbach, in: Plenarprotokoll des Bundesrats, Stenografischer Bericht über die 809. Sitzung, S. 88 A, C; Schewe-Gerigk, in: Plenarprotokoll des Bundestags, Stenografischer Bericht über die 178. Sitzung, S. 16784 C.

112 Vgl. Mertin, in: Plenarprotokoll des Bundesrats, Stenografischer Bericht über die 809. Sitzung, S. 87 A.

113 Pollähne, NK 2002, 56.

114 Hartenbach, in: Plenarprotokoll des Bundestags, Stenografischer Bericht über die 178. Sitzung, S. 16786 A. 
regelmäßige Dauer eines Strafverfahrens von der Anzeige bis zur rechtkräftigen Verurteilung ist kaum dazu angetan, zum wirksamen Schutz von Stalking-Opfern beizutragen, ganz abgesehen davon, dass sie im Falle der regelmäßig zu erwartenden Geld- oder zur Bewährung ausgesetzten Freiheitsstrafen weiterhin darauf vertrauen müssen, dass der Täter sich die Verurteilung zur Warnung dienen lässt (vgl. § 56 Abs. 1 StGB). ${ }^{115}$

Aus Opfersicht erscheint es hinnehmbar, in Fällen von Stalking, in denen die Intensität der einzelnen Handlungen so gering ist, dass eine Strafbarkeit nach allgemeinen Strafvorschriften des StGB nicht gegeben ist, auf dem Zivilrechtsweg gegen den Stalker vorzugehen. Bei wiederholten Verstößen kann das Opfer dann ein Vorgehen gegen den Täter nach dem GewSchG initiieren. ${ }^{116}$

Aus dem Blickwinkel des Strafrechts ist eine Anpassung der Stalking bezogenen Vorschriften des GewSchG an die aktuellen Gegebenheiten - insbesondere die Ausweitung des Katalogs der relevanten Stalking-Handlungen sowie eine Erhöhung des Strafrahmens - geboten aber auch ausreichend.

Trotz der erheblichen Bedenken, die gegen die Schaffung eines eigenen StalkingStraftatbestandes sprechen, dürfte vor dem Hintergrund der erheblichen Popularität des Gesetzesvorhabens die Verabschiedung eines neuen Straftatbestandes zu erwarten sein. Auch in der aktuellen Koalitionsvereinbarung zwischen CDU/CSU und SPD wird das Phänomen Stalking im Bereich Rechtspolitik ausdrücklich angesprochen. Hiernach sollen beharrliche Nachstellungen, die einschneidend das Leben des Opfers beeinträchtigen, in einem eigenen Straftatbestand unter Strafe gestellt werden. ${ }^{117}$ Nachdem sich der Bundesrat Ende September 2005 in einer ablehnenden Stellungnahme im Anschluss an die Empfehlungen der Ausschüsse ${ }^{118}$ zum Gesetzesentwurf der Bundesregierung geäußert und insbesondere die Lückenhaftigkeit des vorgeschlagenen Tatbestandes sowie die Notwendigkeit der im ursprünglichen Entwurf des Bundesrats vorgesehenen Deeskalationshaft zum Ausdruck gebracht hat, ${ }^{119}$ erscheint jedoch ungewiss, welche konkrete Gestalt ein neuer Straftatbestand in der neuen Legislaturperiode annehmen wird. Nachdem sowohl die Bundesregierung ${ }^{120}$ als auch der Bundesrat ${ }^{121}$ Mitte Februar 2006 die Gesetzesentwürfe aus der letzten Legislaturperiode in unveränderter Form in ein neues Gesetzgebungsverfahren eingebracht haben, darf der Weg zur Einigung auf eine Kompromissformel für eine Tatbestandsformulierung mit Spannung beobachtet werden. Inwieweit dieser neue Stalking-Straftatbestand dann den verfassungsrechtlichen Bestimmtheitsforderungen Stand halten wird, bleibt abzuwarten.

115 Pollähne, NK 2002, 56, 58.

116 Zur Abgrenzung des sog. milden und schweren Stalking: Bettermann, in Bettermann/Feenders [Fn. 3] S. 3, 5.

117 Koalitionsvereinbarung CDU/CSU und SPD, Abschnitt 2.1, S. 49. Der Koalitionsvertrag steht auf der Website der Bundesregierung unter http://www.bundesregierung.de im Volltext zum Download bereit.

118 Empfehlungen der Ausschüsse, BR-Drs. 617/1/05 vom 13.09.2005.

119 Stellungnahme des Bundesrats, BR-Drs. 617/05 vom 23.09.2005.

120 Gesetzesentwurf der Bundesregierung, BT-Drs. 16/575.

121 Gesetzesantrag des Landes Baden-Württemberg, BR-Drs. 48/06; Plenarprotokoll des Bundesrats, Stenografischer Bericht über die 819. Sitzung, S. 10 C. 